\title{
Ecoturismo comunitario, una opción de desarrollo local desde la economía inclusiva y la interculturalidad: Sendero ecológico en el Corregimiento de Chorrera, Municipio de Distracción - La Guajira, Colombia
}

\begin{abstract}
Rosa Mercedes Gómez , Navil Mindiola Carrillo², Claudia Giovana Solarte ${ }^{3}$

${ }^{1}$ Universitaria de Popayán, Colombia ${ }^{2}$ Universitaria de Popayán, Colombia

${ }^{3}$ Universitaria de Popayán, Colombia
\end{abstract}

\section{Resumen}

El presente artículo tiene como objetivo visibilizar el ecoturismo comunitario como una opción de desarrollo local desde la economía inclusiva y la interculturalidad. Para ello se tomará en cuenta el caso del sendero ecológico en el corregimiento de Chorrera,

Municipio de Distracción-La Guajira, Colombia. La propuesta está enmarcada

en las dinámicas derivadas del Proyecto de Educación Inclusiva en el Programa de Contaduría Pública de la Fundación Universitaria de Popayán. Es un estudio de carácter descriptivo, con acercamiento al contexto local. Se busca con él que la comunidad se apropie y lidere sus propuestas de desarrollo, la protección del territorio y el posicionamiento de la interculturalidad.

Palabras clave: ecoturismo comunitario, economía inclusiva, interculturalidad, desarrollo local

\footnotetext{
Community Ecotourism: a local development option from an inclusive economy and interculturality: Ecological trail in the Chorreras Corregimiento - Municipality of Distracción - La Guajira- Colombia
} 
Ecoturismo comunitario, una opción de desarrollo local desde la economía inclusiva y la interculturalidad: Sendero ecológico en el Corregimiento de Chorrera, Municipio de Distracción - La Guajira, Colombia

\title{
Summary
}

The objective of this article is to make community ecotourism visible as an option for local development from an inclusive economy and interculturality. For this purpose, the case of the ecological trail in the village of Chorrera, Municipality of DistracciónLa Guajira, Colombia, will be taken into account. The proposal is framed within the dynamics derived from the Inclusive Education Project in the Public Accounting Program of the Fundación Universitaria de Popayán. It is a descriptive study, with an approach to the local context. It seeks for the community to take ownership and lead their development proposals, the protection of the territory and the positioning of interculturality.

Keywords: community ecotourism, inclusive economy, interculturality, local development

\section{Ecoturismo comunitário: uma opção de desenvolvimento local a partir de uma economia inclusiva e interculturalidade: Trilha ecológica no Chorreras Corregimiento - Município de Distracción - La Guajira- Colômbia}

\begin{abstract}
Resumo
Este artigo visa tornar o ecoturismo comunitário visível como uma opção para o desenvolvimento local a partir da economia inclusiva e da interculturalidade. Para este efeito, será tido em conta o caso do trilho ecológico na aldeia de Chorrera, Município

de Distracción-La Guajira, Colômbia. A proposta está enquadrada na dinâmica derivada do Projecto de Educação Inclusiva no Programa de Contabilidade Pública da Fundación Universitaria de Popayán. Trata-se de um estudo descritivo, com uma abordagem ao contexto local. O objectivo é que a comunidade se aproprie e lidere as suas propostas de desenvolvimento, a protecção do território e o posicionamento da interculturalidade.
\end{abstract}

Palavras-chave: ecoturismo comunitário, economia inclusiva, interculturalidade, desenvolvimento local 


\section{Introducción}

Esta propuesta, se llevó a cabo en el marco de los procesos generados en torno al proyecto de Educación Inclusiva desarrollado en el Programa de Contaduría Pública de la Fundación Universitaria de Popayán, en el cual se perfilaron estrategias pedagógicas para trasladar las claves de inclusión y reconocimiento de la diversidad al aula y a los espacios de investigación formativa, en este caso particular al Semillero de Desarrollo e Investigación Social SEDIS. Se trabaja así la línea de Economías Inclusivas como una oportunidad para acercar al programa de Contaduría Pública a otros escenarios en donde las lógicas económicas y contables se configuran desde lo local (Ahumada \& Gómez, 2020) y que derivan de otras miradas epistémicas que se centran en la valoración de lo propio y de la interrelación con los territorios, producto de la cotidianidad de la vida y de la construcción permanente del entretejido entre naturaleza y cultura, consolidándose como una alternativa de participación justa y equitativa, que valora los modelos provenientes de las economías ancestrales y sociales. Es en este contexto de referencia donde surge la posibilidad de abordar, por parte de algunos integrantes del Semillero SEDIS, la temática del ecoturismo como opción de desarrollo local en la región de La Guajira, considerando la procedencia de uno de ellos y su interés en propiciar alternativas que en el mediano y largo plazo contribuyan al bienestar económico y social de la comunidad.

En este orden de ideas y con relación a la iniciativa en mención, se subraya que el departamento de La Guajira es una de las regiones en Colombia que paulatinamente se ha ido posicionando como destino turístico, tanto por la riqueza de su recursos naturales asociados a ecosistemas marinos y desérticos, los bellos paisajes (tales como el Cabo de la Vela, las Salinas de Manaure, las playas de Mayapo, Riohacha, Camarones, entre otros) y la riqueza cultural de comunidades campesinas, afrodescendientes e indígenas (visibilizada por la etnia Wayuu). Este contexto conduce de manera natural a que el turismo sea el motor del desarrollo regional y que se constituya en uno de sus sectores económicos relevantes. El Corregimiento de Chorrera-Municipio de Distracción, se encuentra ubicado paralelo al cauce del Río Ranchería, principal recurso hídrico del departamento, y cerca al área de influencia de la represa Ranchería, condición que ubica a esta zona en una posición estratégica para ser aprovechada desde el ecoturismo comunitario. Por su localización y la riqueza paisajística, se consolida como un escenario para actividades sociales y culturales tradicionales, que pueden potenciarse desde la organización y habilitación del sendero ecológico desde el Sector el Pasito hasta el Cercao, en donde está ubicada la represa, que resalta por ser un espejo de agua de 534 hectáreas con una profundidad en su cota más alta de 105 metros.

Se visibiliza que el ecoturismo comunitario se puede configurar como una opción de desarrollo local para el corregimiento, en la medida en que le apueste a la consolidación de modelos sostenibles. Al ser un sitio privilegiado, permite la estructuración de propuestas que promuevan el uso adecuado de los recursos naturales y la preservación de los ecosistemas estratégicos de la zona; así como la generación de capacidad instalada económica y socialmente. Se hace énfasis que la finalidad es apostarle a un turismo alternativo "que respeta el medio ambiente 
Ecoturismo comunitario, una opción de desarrollo local desde la economía inclusiva y la interculturalidad: Sendero ecológico en el Corregimiento de Chorrera, Municipio de Distracción - La Guajira, Colombia

y donde el turista adquiere nuevas experiencias en contacto con la naturaleza, la cultura y la población local del destino turístico" (Orgaz \& Cañero, 2015, p. 49); lo anterior en aras de evitar conflictos que vayan en detrimento de la población local y posibiliten el empoderamiento económico y social de la población asentada en una región.

\section{Marco Teórico}

Acerca de la conceptualización sobre el ecoturismo, la primera definición la da la Sociedad Internacional de Ecoturismo en 1990. Hace alusión al "viaje responsable a zonas naturales que conserva el medio ambiente y mejora el bienestar de las poblaciones locales" (Drumm \& Moore, 2002, p.16). Una definición más estructurada la proporciona Jalani (2012 citado en Orgaz \& Cañero 2015), quien menciona que el ecoturismo hace referencia a aquel tipo de turismo que se desarrolla en áreas naturales y tiene como objetivo la conservación de las zonas naturales y el desarrollo de las comunidades locales. De manera complementaria, Ramírez (2014) expresa que se trata de una modalidad que combina la pasión por el viaje con la preocupación por el ambiente.

En cuanto a los componentes que conforman la actividad ecoturística, Orgaz y Cañero (2015) mencionan que el ecoturismo lo componen aquellas actividades que entran en contacto con la naturaleza y sus destinos se asocian hacia áreas naturales protegidas y/o sitios con una belleza natural e interés ecológico.

En lo que concierne al turismo comunitario, Cabanilla (2018) manifiesta que es un término en auge, pero a su vez en construcción, que se alimenta de las investigaciones que van surgiendo y que alude a un modelo de gestión cuya finalidad es la generación de impactos económicos positivos distribuidos entre los miembros de una comunidad. Este mismo autor resalta en los orígenes del concepto: la Declaración de la OMT dada en Manila en 1980. En ella se recoge la demanda de varios territorios que se consideran desplazados por el modelo de planificación turística que hasta la fecha se llevaba a cabo, situación que da origen a una serie de artículos y manuales que dan lineamientos a los planificadores turísticos para trabajar el turismo desde lo local.

En varios países latinoamericanos, resalta Cabanilla (2018), el concepto de turismo comunitario se alimentó desde autores principalmente norteamericanos, que lo vincularon con los de turismo rural o de base local, apalancados desde una política que impulsa el turismo en comunidades rurales, como una herramienta potencial para su desarrollo. En su estudio, Cabanilla (2018) cita autores como Page \& Getz (1997), Butler, Hall \& Jenkins (1998), Pearce, Moscardo \& Ross (1996), entre otros, que apalancaron desde sus postulados el fortalecimiento de este concepto.

Es Maldonado (2005) quien proporciona tal vez la primera definición de turismo comunitario en la que se entrevé una solidez y una finalidad, que permite la aproximación desde la academia: "es toda forma de organización empresarial 
sustentada en la propiedad y la autogestión de los recursos patrimoniales comunitarios; contribuye a la solidaridad en el trabajo y distribución de los beneficios generados por la prestación de servicios turísticos" (p. 5). Burgos (2016) plantea que el turismo comunitario surge como una forma de autogestión de los recursos patrimoniales de una comunidad con el fin de aportar a procesos de solidaridad en el trabajo, pero también como una alternativa de desarrollo local, ya que a través de esta actividad las comunidades no solo generan ingresos y mejoran su calidad de vida, sino que configuran mecanismos para preservar su patrimonio natural y cultural.

A nivel de Latinoamérica, se destacan distintos trabajos, como el de CamachoRuiz, Carrillo-Reyes, Rioja-Paradela \& Espinoza-Medinilla (2016), orientado a la construcción de indicadores para un manejo más sostenible del turismo y su articulación a comunidades locales en México. Márquez (2015) estudia la vernacularización de la conservación en los territorios indígenas amazónicos de Bolivia, buscando comprender cómo mediante estos dispositivos de generación de ingresos económicos locales de "bajo impacto ambiental", los principios de la conservación y el desarrollo sostenible intentan ser traducidos a las formas de vida de dos pueblos amazónicos. Y Orgaz y Cañero (2015) se aproximan al ecoturismo como motor de desarrollo en zonas rurales; y a través del estudio de caso en República Dominicana, buscan entender los beneficios económicos, socioculturales y medioambientales percibidos por el ecoturismo por parte de los residentes de comunidades rurales.

Para el caso de Colombia, se resaltan los estudios de Huertas-Cardozo (2016), con su propuesta de innovación social y turismo rural comunitario en los Montes de María. También los de Ramírez (2014) sobre el ecoturismo y su incidencia en el desarrollo regional en Santander y Quindío; y Burgos (2016), en Usme y Ciudad Bolívar, para posicionar estas localidades como destinos para el turismo comunitario. De igual manera tenemos los artículos de Cujía, Pérez y Maestre (2017), con el papel que juega el ecoturismo en el desarrollo regional y su articulación al conocimiento científico y el uso de la tecnología, desde la transferencia a las comunidades locales; y Ochoa, James y Márquez (2013), acerca del proceso llevado en el Parque Nacional Natural Amacayacu, con las comunidades indígenas. Finalmente, para el caso específico de La Guajira, tenemos el trabajo de Mejía (2015), sobre caracterización de ofertas turísticas.

En cuanto al concepto de desarrollo local, para efectos de este trabajo, se privilegia la definición dada por Lorenzo y Morales (2014), quienes enfatizan en que es un proceso que se fortalece desde "la integración socio económica y medioambiental de todos los factores que forman parte de una región o localidad, donde los agentes locales tras el uso de los recursos tanto endógenos como exógenos son capaces de crear mejora continua en su calidad de vida" (p. 454).

De manera complementaria, Di Pietro (2014), apunta que el desarrollo local, es la respuesta de localidades y regiones a un desafío de carácter global, que apunta a procesos de desarrollo endógeno, en otras palabras el impulso a la capacidad 
Ecoturismo comunitario, una opción de desarrollo local desde la economía inclusiva y la interculturalidad: Sendero ecológico en el Corregimiento de Chorrera, Municipio de Distracción - La Guajira, Colombia

emprendedora local, que pueda de alguna manera hacer frente a las políticas macroeconómicas que tienen como resultados procesos de distribución inequitativa que se derivan del enfoque de crecimiento económico, desconociendo otras esferas. De lo anterior se desprende que el desafío está en articular las dimensiones económica y social.

En lo que respecta a la noción de economía inclusiva, ésta se asume como aquel modelo económico que promueve la participación en el sistema económico de todos los integrantes de un contexto social en el marco de la justicia y la equidad. Este es un concepto aún reciente en el ámbito académico y es muy poca la literatura existente sobre el mismo. Álvarez, Barceló, Carpintero, Carrasco, Martínez, Recio y Roca (2012), abordan este concepto como un paradigma sistémico a partir del cual se puede asumir una posición crítica frente al capitalismo y desde allí generar enfoques alternativos para interpretar la realidad social y hacer frente a la crisis multidimensional que caracteriza el momento actual. Lo anterior supone generar una representación de la realidad económica, desde las diferentes dimensiones que en ella intervienen y desde enfoques complementarios como la economía ecológica y la economía política. En otras palabras, se plantea la necesidad de generar un enfoque, que se nutra de los postulados de la economía crítica, pero que a su vez la trascienda (Martínez, 2017). Pastor y Giusta (2017) hablan de la expansión de oportunidades para una prosperidad ampliamente compartida, especialmente para aquellos que enfrentan mayores barreras para avanzar su bienestar socioeconómico; contemplan cinco características interrelacionadas: equidad, participación, crecimiento, sostenibilidad y estabilidad.

En cuanto al concepto de Interculturalidad, cabe anotar que surge como perspectiva en América Latina principalmente desde el discurso y las praxis de las comunidades indígenas, colocando la puesta en común de valores, perspectivas, ideologías e intereses, que permiten "la consolidación de un proyecto político, social, epistémico y ético dirigido a la transformación cultural y socio-histórica. Esta hace entonces referencia al cruzamiento, la relación, el contacto, el mestizaje, la confrontación, la negociación, la intersección, interpenetración o inter estructuración positiva de culturas" (Castillo \& Guido, 2015, p.19).

\section{Metodología}

A partir de lo anterior, nos planteamos a partir de ahora plantear la metodología del trabajo. Se trata de un estudio descriptivo, con énfasis cualitativo, que apunta a la caracterización ambiental y sociocultural, focalizados en los valores ecológicos y culturales en el área del Río Ranchería, sector del Corregimiento de Chorrera, por donde se prospecta el sendero. Se hará uso de la recopilación documental, los recorridos de reconocimiento del área y las entrevistas semiestructuradas con los propietarios de los 28 predios, docentes de la IER Gladys Bolilla de Gil, el alcalde de Distracción Yesid Peralta Suarez y Luis Gustavo Daza Secretario de Planeación Municipal, además con José Prudencio Nieves y Armando Añez, funcionarios de 
la Corporación Autónoma Regional de La Guajira, con el propósito de conocer su percepción sobre esta iniciativa y las ventajas económicos y sociales que a mediano plazo podría tener si se lleva a cabo en su totalidad. También se adelanta la proyección inicial de los costos en torno a la organización y habilitación del sendero, prospectada en primer lugar al tema de las obras físicas del mismo, y en segundo lugar a la identificación de costos ambientales relacionados con procesos de recuperación de áreas ecológicas vulnerables con el fin de abrir el escenario a corto plazo hacia la generación de capacidad instalada en la comunidad para la prestación de servicios ecoturísticos comunitarios.

\section{Resultados y Discusión}

\section{Figura 1: Represa Río Ranchería}

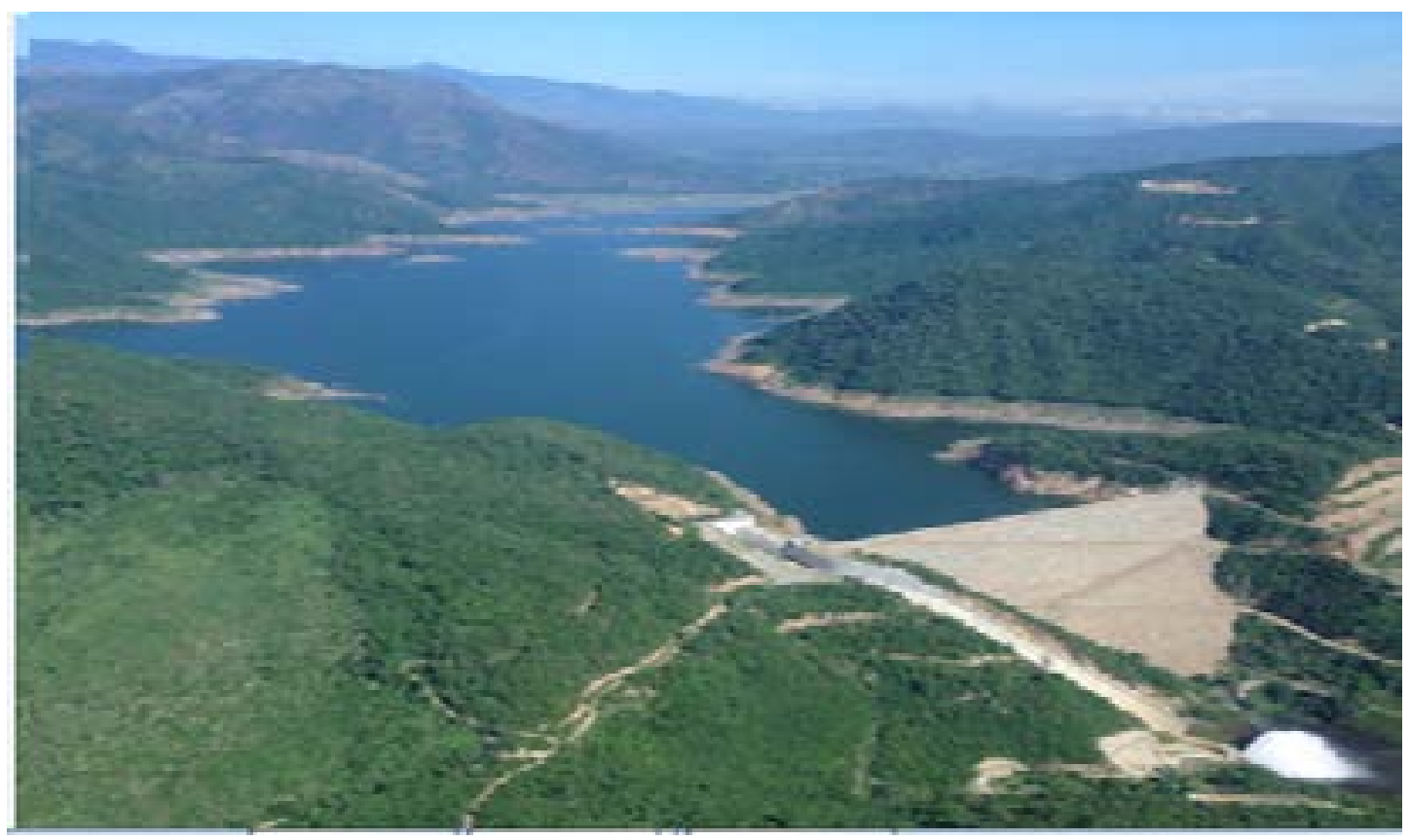

Fuente: Gutiérrez (2017)

Esta propuesta se lleva a cabo en el Municipio de Distracción, ubicado en la zona baja del departamento de la Guajira, con una gran extensión de tierras planas y topografía irregular, que se nutre de los arroyos provenientes del norte y que drenan al río Ranchería, principal afluente de la región (Gobernación de La Guajira, 2016, p. 3). Corresponde al piedemonte de la Sierra Nevada de Santa Marta, la Serranía del Perijá y los Montes de Oca, con gran variedad de microclimas y tierras cultivables. Es allí, en el corregimiento de Chorrera, donde se ubica la represa del Río Ranchería, que abastece gran parte del departamento. Se destacan los relictos de bosque, por su potencial biológico para la regeneración y restauración de los ecosistemas colindantes, así como la diversidad de fauna y flora asociada (Ministerio de Comercio, Industria y Turismo, s/f, p. 22-23).

En cuanto a lo social, se destaca en su historia, que el territorio estuvo ocupado 
Ecoturismo comunitario, una opción de desarrollo local desde la economía inclusiva y la interculturalidad: Sendero ecológico en el Corregimiento de Chorrera, Municipio de Distracción - La Guajira, Colombia

antes de la llegada de los españoles por grupos indígenas que habitaron cerca al río, en las estribaciones de la Sierra Nevada, los Arahuacos y los Wayuu, en la orilla del Río Ranchería. Inicialmente, Distracción pertenecía a Fonseca como corregimiento y fue elevado a municipio en 1995 (Gobernación de La Guajira, 2016, p. 4).

La región cuenta con 15.790 habitantes (Gobernación de La Guajira, 2016, p.6). Para el caso específico del Corregimiento de Chorrera, los registros de la Alcaldía Municipal a 2020, dan cuenta de 282 familias, aproximadamente 1500 habitantes. En el corregimiento se encuentran asentamientos campesinos e indígenas. Estas últimas se encuentran asentados en el Sector de El Chorro, Resguardo de Potrerito ${ }^{1}$ (comunicación personal, 2 de enero, 2020).

En lo cultural, la región sur se destaca la pasión por el ritmo vallenato, cuna de acordeones y juglares, en donde el ritmo emana de la combinación melodiosa de la caja, la guacharaca y el acordeón, compositores y decimeros, varios de ellos oriundos del corregimiento ${ }^{2}$.

\section{El área del sendero}

El sendero posee un total de 14 kilómetros desde el Sector el Pasito hasta el Cercao, en donde está ubicada la represa, que resalta por ser un espejo de agua de 534 hectáreas con una profundidad en su cota más alta de 105 metros. Sobre el área hay 29 predios identificados.

\section{Figura 2: Recorrido hacia la Represa del Río Ranchería}

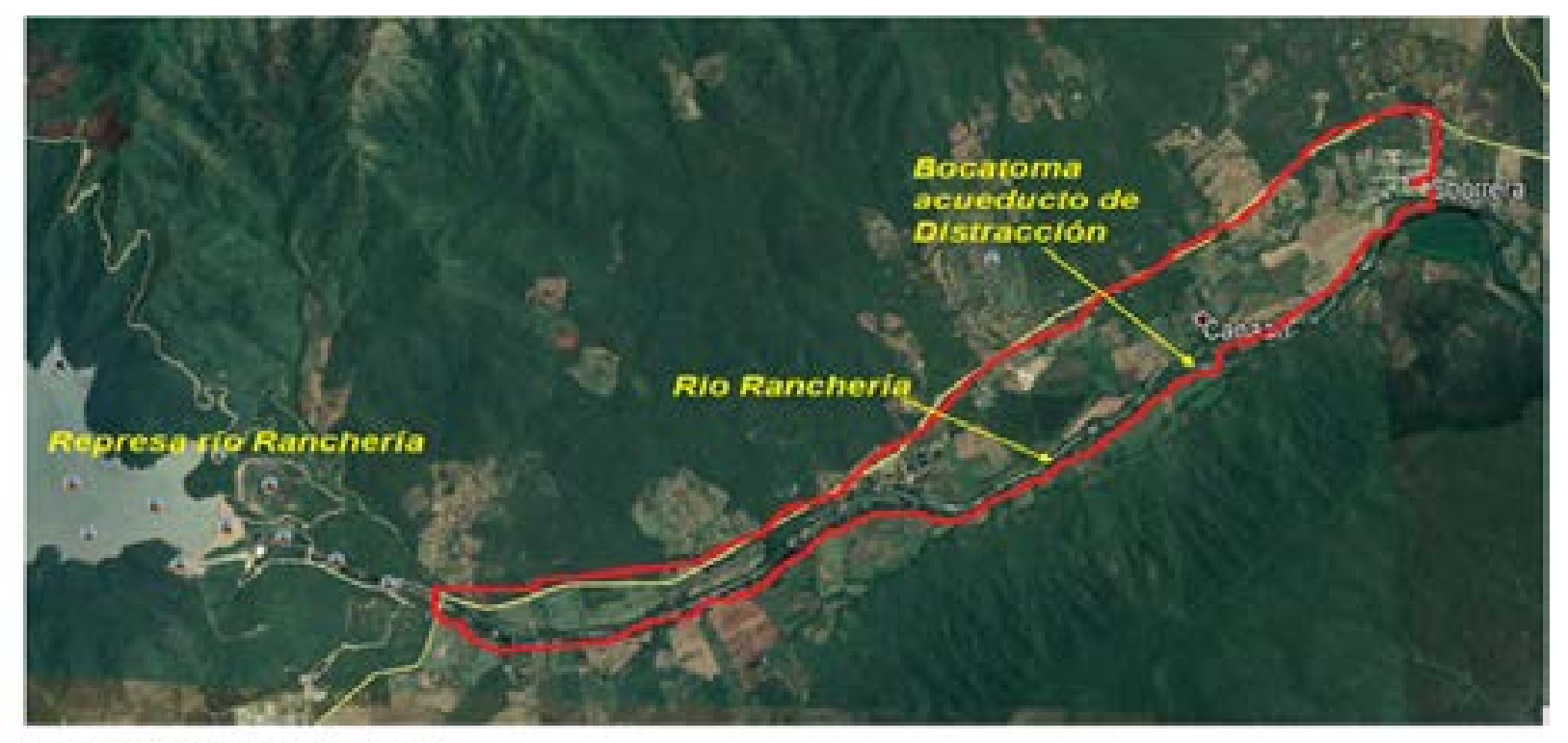

Fuente: : Elaboración Propia con apoyo de Google Maps.

1 Información obtenida de la Secretaría de Planeación.

2 El escritor José Ceferino Nieves, gran conocedor de la historia y cultura local, destaca acordeoneros como Moisés Daza "Miché", Pedro Pablo Arocha, Moisés y Calixto Daza Mejía, Ramón Mejía, Ernesto y Alonso Gil Carrillo, guitarristas como Gerardo Molina, Erick Orozco, José María Gil Brito y Osmelin Mejía; compositores como Freddy Carrillo, Erick Orozco, Numas Molina, Arnold Nieves, Enrique Brito Mejía, Lermith Daza Carrillo y Osmelin Mejía, decimero como Rafael Darío Alvarado 
El reconocimiento del área, se hizo con la autorización y acompañamiento de los propietarios, para recoger sus apreciaciones frente a la propuesta, identificar potencialidades ecoturísticas y hacer el levantamiento de la fauna y flora existente. En las entrevistas realizadas de manera informal, los propietarios consideran que la habilitación del sendero puede ser una oportunidad para reactivar la economía de la región, ya que este camino ha sido utilizado para el ciclo-montañismo y el senderismo principalmente en los fines de semana, pero no de manera estructurada y formal, ni considerando la apertura de nuevas actividades y prestación de servicios asociados como el hospedaje, la alimentación y la guianza.

Se aborda en el marco de estas conversaciones, la posibilidad de diversificar ingresos, ya que a mediano plazo las fincas se podrían proyectar procesos de visitancia y así visibilizar las actividades que dentro de ellas se realizan, generando ganancias adicionales a las actividades agropecuarias que les son propias. Adicionalmente, se habla de la importancia de fortalecer procesos asociativos, que permitan la comercialización directa de los productos y abolir el tema de los intermediarios y la organización de un centro de acopio un día a la semana que funcione como mercado satélite. Se destacan a continuación algunas voces de los propietarios:

"Teniendo en cuenta, lo que usted dice, la idea sería mostrar a los visitantes, no solo la ida hasta la represa, que es muy linda, sino también, los cultivos y productos de la zona" (Rolfin Carrillo, comunicación personal, 4 de enero del 2020).

"Uno podría contarles a los visitantes, como es que se cultiva el arroz, que es tan propio de acá, que ellos vean el proceso de pilado y que participen de varios de nuestros procesos, que aún se hacen de manera manual y así con otros productos" (Riquelmis Caicedo, comunicación personal, 30 de diciembre del 2019).

Al respecto de este proceso, el rector de la institución educativa de la población añade: "La idea es buena, porque sería con gente de acá y no de afuera, ganarían en el pueblo también porque allí se movería el tema de las comidas y posada y para acá en las fincas los recorridos y el mostrar nuestros productos... " (Numas Molina Comunicación personal, 30 de diciembre del 2019).

Llama la atención de esta propuesta la posibilidad de que las mujeres y jóvenes generen actividades complementarias que les permitan aumentar sus ingresos y ayudar a la economía doméstica. Gran parte de ellas se desenvuelven en torno a las labores del hogar y acompañamiento de actividades dentro de las fincas, por lo que una oportunidad como la que aquí se plantea les permitiría explorar en otros ámbitos. Los jóvenes, desde la posibilidad de ser económicamente activos, evitando procesos de migración hacia la ciudad: "Se imagina, uno podría hacer artesanías aquí en la casa... hacer dulces y comidas de acá, para que el turista conozca, darles posada y atenderlos y así uno se hace una entradita" (Jacob Brito, comunicación personal, 3 de enero, 2020). Otro de los jóvenes señala: "Cuando uno sale del colegio, muchas veces no sabe qué hacer ni pa' dónde coger, así uno podría hacer otras actividades y quedarse por acá, también salir a estudiar pero pensando siempre en volver a aportar acá a su gente" (Juan José Mendoza, comunicación personal, 30 de diciembre, 2019). 
Ecoturismo comunitario, una opción de desarrollo local desde la economía inclusiva y la interculturalidad: Sendero ecológico en el Corregimiento de Chorrera, Municipio de Distracción - La Guajira, Colombia

Un factor clave, es la coexistencia en el territorio de comunidad campesina e indígena, lo que puede conllevar a la apertura de nuevos escenarios que, desde la interculturalidad, permitan consolidar propuestas económicas alternativas en común, en donde ambos sectores salgan beneficiados, no solo desde la visibilización de sus territorios en cuanto a lo paisajístico, sino desde la promoción de sus valores culturales materiales e inmateriales.

Desde la Administración Municipal actual y la Corporación Autónoma Regional de La Guajira33, se evidencia voluntad política, para apoyar este tipo de iniciativas, que conducen al aprovechamiento sostenible de la oferta ambiental y cultural de la zona y que a su vez operan como catalizadoras para impulsar el desarrollo propio (comunicación personal, 2 de enero,2020). Se revisó con ellos el informe preliminar de los recorridos para ver las obras físicas y de carácter ambiental que deben entrar a considerarse; algunas de ellas ya se incorporaron en el plan de desarrollo municipal, las otras son susceptibles de ser gestionadas sin mayor inconveniente. Igualmente, se abordó el tema económico para posibilitar acciones que conduzcan al fortalecimiento del sector productivo, considerando la vocación agropecuaria del municipio y que se abordaron en las conversaciones con los propietarios de los predios que están ubicados en el área del sendero. Un factor a considerar en el mediano plazo, es el proceso de formación que debe llevarse a cabo con la comunidad, para la adecuada prestación de los servicios ecoturísticos asociados a hospedaje, alimentación, guianza y comercialización, para lo cual se requiere el apoyo de la administración municipal y las demás instituciones con injerencia en el tema.

La socialización de esta propuesta, también se llevó a cabo con los docentes de la Institución Educativa "Gladys Bonilla de Gil" misma, ya que involucra el turismo, como un elemento dinamizador de la economía local, con el valor agregado de que la comunidad es el actor principal. En este sentido, resaltan que se podría pensar en la articulación de proyectos escolares y de educación continuada que respalden esta iniciativa y que se orienten a aspectos relevantes como: educación ambiental, economía solidaria, transformación de productos, gestión de recursos naturales, artesanías, gestión cultural, entre otros que podrían entrar a ser apoyados desde la administración municipal, la corporación Autónoma Regional de la Guajira (comunicación personal, 3 de enero, 2020). Es una oportunidad a su vez, para dinamizar los contenidos curriculares hacia los bienes ambientales y culturales existentes en la región, de tal manera que se propicie el sentido de pertenencia hacia la misma y la valoración de lo propio.

Se identificaron a lo largo de los recorridos las siguientes mejoras y adecuaciones a realizaren el sector:

- Puente sobre el arroyo Media Luna, a 100 metros del inicio del recorrido. Se

3 Información recogida a partir de entrevista con el Alcalde Municipal Yesid Peralta Suarez y José Prudencio Nieves funcionario de la Corporación Autónoma Regional de la Guajira.

4 Numas Molina, Edmundo Mejía, Enilda González, Asleys Murillo Eider Oñate, Orley Rodríguez, Belkis Mendoza, Sandra Mendoza, Leibeth Mendoza 
requiere construir un box coulvert doble de $2 \times 2$ metros que ayudara a mejorar el carreteable y disminuir la pendiente.

- Box coulvert sobre el arroyo de la Toña. Se debe construir el box coulvert para mejorar el tránsito por el carreteable; se trata de un cuadrado de $1 \times 1$ metro.

- Drenaje sobre el arroyo El Ranchito. En este punto el agua se represa y es un tramo de unos 50 metros antes de verter sus aguas la rio Ranchería.

- Apertura de camino real. Se requiere contratar un aserrador para que con moto sierra pueda cortar o trozar el árbol para permitir el paso en el mismo.

- En cuanto al componente ambiental, se hace necesario efectuar algunas actividades de mejoramiento del área, debido a que en el mes de diciembre de 2019, un vendaval derribó árboles grandes y parte de la vegetación nativa. Se propone llevar a cabo actividades de reforestación con la siembra de 150 árboles nativos. De igual manera las campañas de sensibilización y concientización y el mantenimiento del sendero.

La proyección de costos sería la siguiente:

\section{Tabla 1. Obras Físicas}

\begin{tabular}{|l|l|}
\hline ITEM & VALOR TOTAL (COP) \\
\hline $\begin{array}{l}\text { Box coulvert } 2 \times 2 \text { metros en } \\
\text { el arroyo Media Luna. }\end{array}$ & $\$ 58.788 .267$ \\
\hline $\begin{array}{l}\text { Box coulvert } 1 \times 1 \text { metro en } \\
\text { el arroyo La Toña }\end{array}$ & $\$ 23.920 .010$ \\
\hline Drenaje arroyo El Ranchito & $\$ 150.000$ \\
\hline SUBTOTAL & $\mathbf{\$ 8 2 . 8 6 7 . 2 7 7}$ \\
\hline
\end{tabular}

Fuente: Elaboración Propia 
Ecoturismo comunitario, una opción de desarrollo local desde la economía inclusiva y la interculturalidad: Sendero ecológico en el Corregimiento de Chorrera, Municipio de Distracción - La Guajira, Colombia

\section{Tabla 2. Aspectos ambientales}

\begin{tabular}{|l|l|}
\hline ITEM & VALOR TOTAL (COP) \\
\hline $\begin{array}{l}\text { Reforestación, especies } \\
\text { nativas }\end{array}$ & $\$ 300.000$ \\
\hline Mano de obra & $\$ 500.000$ \\
\hline Insumos & $\$ 225.000$ \\
\hline Mantenimiento 1 año & $\$ 1.060 .000$ \\
\hline $\begin{array}{l}\text { Valla informativa 3x2 mts } \\
\text { madera plástica }\end{array}$ & $\$ 1.350 .000$ \\
\hline 70 letreros 35x25 cms & $\$ 1.575 .000$ \\
\hline Campañas de sensibilización & $\$ 100.000$ \\
\hline Aserrador & $\$ 5.710 .000$ \\
\hline
\end{tabular}

Fuente: Elaboración Propia

\section{Tabla 3. Costos Totales}

\begin{tabular}{|l|l|}
\hline ITEM & VALOR TOTAL (COP) \\
\hline Obras Físicas & $\$ 82.867 .277$ \\
\hline Aspectos ambientales & $\$ 5.710 .000$ \\
\hline TOTAL & \\
\hline
\end{tabular}

Fuente: Elaboración Propia 


\section{Avanzando en el análisis}

Como ya se mencionó arriba, esta propuesta se enfocó en la posibilidad de posicionar el ecoturismo comunitario como una opción de desarrollo local en el corregimiento de Chorreras, y como una opción que permita un giro a las dinámicas económicas de la región. El propósito inherente es lograr el empoderamiento de los actores locales, el despliegue de su capacidad de agencia y el mejoramiento de su calidad de vida. Considerando que el turismo que actualmente se estimula a nivel mundial es aquel en el cual la población local participa de su planificación, gestión y promoción (Burgos, 2016), se aspira a que la comunidad del Corregimiento de Chorrera, especialmente los propietarios de los predios ubicados en el área del sendero, rompan las barreras al cambio y vean en estos procesos, una oportunidad de participar en ellos desde el inicio, apuntando a la protección del territorio y de la interculturalidad que allí se da.

Es necesario que el desarrollo de la propuesta involucre la autogestión, la solidaridad y la distribución equitativa y justa de los beneficios que se deriven en el mediano y largo plazo de la prestación de los servicios ecoturísticos. Como lo menciona Burgos (2016), este tipo de oportunidades contribuye con el desarrollo de las comunidades y les proporciona ingresos adicionales que contribuyen al mejoramiento de su calidad de vida.

Se busca, en este caso específico, que los pobladores que se encuentran especialmente en el área del sendero ecológico puedan consolidar la diversificación de sus ingresos, desde emprendimientos locales en los que se promueva la visitancia a sus fincas con el propósito de visibilizar sus procesos productivos tradicionales y actividades derivadas, que a su vez permiten resaltar la riqueza cultural de la región en la que convergen comunidades campesinas e indígenas. Por consiguiente es una oportunidad, para configurar dinámicas económicas inclusivas, respaldadas en la solidaridad, la reciprocidad y el diálogo intercultural, que se apartan de la lógica del mercado y privilegian lo propio (Álvarez et al., 2012). Este ejercicio, fue una oportunidad para compartir y conocer las percepciones de los pobladores locales y las instituciones, para generar lecturas de contexto y reconocimiento del territorio, así como la validación de sus praxis socioeconómicas y culturales, como un punto de partida para avanzar en procesos de autogestión y empoderamiento económico.

Pensar este tipo de dinámicas, en el corregimiento de Chorrera, en donde se encuentran comunidad campesina e indígena, vinculadas al territorio y con elementos dinamizadores como la naturaleza y la cultura, puede contribuir a la constitución de espacios de diálogo e interculturalidad en el que se consoliden propuestas que vayan en beneficio mutuo.

Desde lo académico, es la posibilidad en el programa de Contaduría Pública de avanzar en procesos de fortalecimiento de la investigación formativa, desde tópicos distintos al enfoque instrumental de la formación contable, para dar paso al acercamiento a otros escenarios de validación de las lógicas económicas y contables que provienen de los territorios y que permiten una lectura más social (Ahumada \& Gómez, 2020). Lo anterior, en aras de dinamizar el pensamiento crítico en los 
Ecoturismo comunitario, una opción de desarrollo local desde la economía inclusiva y la interculturalidad: Sendero ecológico en el Corregimiento de Chorrera, Municipio de Distracción - La Guajira, Colombia

estudiantes y permitirles valorar sus contextos y epístemes locales, aperturando espacios de interacción económico-social respaldadas desde la dimensión socio humanística (Ahumada \& Gómez, 2020).

\section{A manera de conclusión...}

Esta apuesta investigativa busca promover, para el Corregimiento de Chorrera, un modelo de gestión de base local, desde el impulso del renglón ecoturístico con carácter comunitario, para posicionar gradualmente dinámicas en las que sea la comunidad la beneficiaria en cuanto a generación y percepción de ingresos, De esa manera, se apuesta por el empoderamiento desde dinámicas económicas inclusivas que propicien el desarrollo local.

En el corregimiento, al tener carácter comunitario y estar orientado hacia la población campesina e indígena, se puede entrever la filiación hacia el territorio, convirtiéndolo en un elemento trascendental para la implementación exitosa de este tipo de alternativas en la región. Especialmente, el área del sendero hacia la represa del río Ranchería se convierte en una oportunidad para gestar dinámicas económicas y socioculturales alternativas.

Así como el ecoturismo comunitario se nutre de los conceptos de naturaleza y cultura, y valora la oferta ambiental y cultural, de igual manera se busca ello con el corregimiento de Chorrera. En este espacio se busca fortalecer la apropiación hacia el territorio, resaltando los recursos naturales y culturales asociados a él, y se propone una relación distinta con el visitante.

Por su carácter comunitario, este tipo de turismo respalda diferentes tipos de emprendimientos y propende por una distribución de ingresos más equitativa, buscando el mejoramiento de la calidad de vida de la población, desde los principios de solidaridad y reciprocidad.

\section{Referencias biliográficas}

Ahumada, M.\&Gómez, M. (2020). Enriqueciendo los procesos de formación contable. Aportes desde las estrategias deeducación inclusiva y la dimensión sociohumanística. Revista Plumilla Educativa, 25 (1), 51-69. DOI: 10.30554/pe.1.3829.2020. Recuperado de https://revistasum.umanizales.edu.co/ojs/index.php/plumillaeducativa/article/ view/3829

Álvarez, S., Barceló, A., Carpintero, O., Carrasco, C., Martínez, Á., Recio, A., \& Roca, J. (2012). Por una economía inclusiva. Hacia un nuevo paradigma sistémico. Revista de Economía Crítica, (14), 277-301. Recuperado de http://revistaeconomiacritica.org/ node/636

Burgos, R. (2016). El turismo comunitario como iniciativa de desarrollo local. Caso localidades de Ciudad Bolívar y Usme zona rural de Bogotá. Hallazgos, 13(26). 
Recuperado de https://doi.org/10.15332/s1794-3841.2016.0026.08

Cabanilla, E. (2018). Turismo comunitario en América Latina, un concepto en construcción. Siembra, 5(1), 121-131. Recuperado de https://doi.org/10.29166/ siembra.v5i1.1433

Camacho-Ruiz, E. Carrillo-Reyes, A., Rioja-Paradela, T., \& Espinoza-Medinilla, E. (2016). Indicadores de sostenibilidad para el ecoturismo en México: estado actual. LiminaR, 14(1), 156-168. Recuperado de http://www.scielo.org.mx/scielo.php?script=sci_ arttext\&pid=S1665-80272016000100011\&lng=es\&tlng=es.

Castillo, E., \& Guido, S. (2015). La interculturalidad: ¿principio o fin de la utopía?. Revista Colombiana de Educación, (69), 17-44. Recuperado de http://www.scielo.org. co/scielo.php?script=sci_arttext\&pid=S0120-39162015000200002\&lng=en\&tlng=.

Cujía, E., Pérez, S., \& Maestre, D. (2017). ECOTURISMO, EDUCACIÓN, Ciencia Y Tecnología, Factores De Desarrollo Sustentable: Caso La Guajira, Colombia. Educación y Humanismo, 19(32). Recuperado de https://doi.org/10.17081/eduhum.19.32.2540

Di Pietro, L. (2014). Hacia un desarrollo rural integrador y equitativo: una introducción al desarrollo local. Recuperado de http://municipios.unq.edu.ar/modules/mislibros/ archivos/555555.pdf

Drumm, A. \& Moore, A. (2002). Desarrollo del Ecoturismo: Un manual para los profesionales de la conservación. Introducción a la planificación del ecoturismo. Virginia: The Nature Conservancy. Recuperado de https://www.researchgate. net/publication/263046518_Desarrollo_del_Ecoturismo_Un_manual_para_ los_profesionales_de_la_conservacion'_Volumen_I_-_Introduccion_a_la_ planificacion_del_ecoturismo

Gobernación de La Guajira, Departamento Administrativo de Planeación (2016). Plan de desarrollo del departamento de La Guajira 2016-2019. Riohacha: Gobernación de La Guajira. Recuperado de https://www.laguajira.gov.co/web/attachments/ article/3371/Plan\%20de\%20Desarrollo\%20La\%20Guajira\%202016-2019\%20-\%20 Parte\%201\%20De\%205.pdf

Gutiérrez, M. (2017). Fotografía de la Represa del Río Ranchería.

https://www.google.com/maps

uv?pb=!1s0x8e8b1e8b8914dad5\%3A0xa0b26cce656421b7!3m1!7e115!4shttps\% 3A\%2F\%2Flh5.googleusercontent.

com\%2Fp\%2FAF1QipNavph1TWVjEN8EWup0D-8-_Yy-ZRprw7MDuvMr\%3Dw213h160-k-no!5srepresa\%20rio\%20rancheria\%20-\%20Buscar\%20con\%20

Google!15sCglgAQ\&imagekey=!1e10!2sAF1QipMu1F0nEdHetxgYKrLTxET9G2PMSwZLYIQV06Az\&hl=es-419\&sa=X\&ved=2ahUKEwjQnKrZ-6bwAhUKU98KHWr_ BMsQoiowJXoECEcQAw

Huertas, N. (2016). Turismo rural comunitario como una propuesta metodológica de innovación social para comunidades en conflicto. Caso Montes de María (Tesis de doctorado, Universitat de Girona, Gerona, España). Recuperado de http://www.tdx. cat/handle/10803/378657 
Ecoturismo comunitario, una opción de desarrollo local desde la economía inclusiva y la interculturalidad: Sendero ecológico en el Corregimiento de Chorrera, Municipio de Distracción - La Guajira, Colombia

Juárez, G. (2013). Revisión Del Concepto De Desarrollo Local Desde Una Perspectiva Territorial. Revista Lider, 23, 9-27. Recuperado de http://ceder.ulagos.cl/lider/images/ numeros/23/1.-LIDER 23_Juarez_pp9_28.pdf

Lorenzo, H. \& Morales, G. (2014). Del desarrollo turístico sostenible al desarrollo local. Su comportamiento complejo. PASOS Revista De Turismo Y Patrimonio Cultural, 12(2), 453-466. Recuperado de https://doi.org/10.25145/j.pasos.2014.12.033

Maldonado, C. (2005). Pautas metodológicas para el análisis de experiencias de turismo comunitario. Ginebra: Organización Internacional del Trabajo [OIT]. Recuperado de http://oit.org/wcmsp5/groups/public/---ed_emp/---emp_ent/---ifp_seed/ documents/publication/wcms_117525.pdf

Márquez,J. (2015). La vernacularización de la conservación en los territorios indígenas amazónicos de Bolivia. Un análisis de la revalorización ancestral indígena en dos proyectos de ecoturismo comunitario en áreas protegidas. Boletín De Antropología, 29(48), 92-118. Recuperado de https://revistas.udea.edu.co/index.php/boletin/ article/view/22216

Martínez, A. (2017). Construir una Economía Inclusiva e integradora. s/c: Foro Transiciones. Recuperado de https://forotransiciones.org/wp-content/uploads/ sites/51/2017/05/Econom\%C3\%ADa-inclusiva.pdf

Mejía, L. (2015). La calidad de las ofertas turísticas en el departamento de la Guajira-Colombia. Dimensión Empresarial, 12(1), 139. Recuperado de https://doi. org/10.15665/rde.v12i1.76

Ministerio de Comercio, Industria y Turismo. Guía Turística: La Guajira, Colombia. Recuperado de https://www.mincit.gov.co/CMSPages/GetFile. aspx?guid=d59284c6-af22-4a03-9996-b94d616e050c

Ochoa, F., James, J. \& Márquez, G. (2013). Visión comunitaria de los beneficios derivados del ecoturismo en el Parque Nacional Natural Amacayacu (Amazonas, Colombia) Gestión y Ambiente, 16 (1), 17-31. Recuperado de http://www.redalyc.org/ pdf/1694/169427489006.pdf

Orgaz F. \& Cañero, P. (2015). El ecoturismo como motor de desarrollo en zonas rurales: un estudio de caso en República Dominicana. Ciencia y sociedad, 40(1), p. 47-76. Recuperado de http://repositoriobiblioteca.intec.edu.do/handle/123456789/1601

Pastor, M. \& Giusta, G. (2017) Economías Inclusivas: Colombia [PPT]. Recuperado de http://inclusiveeconomies.everettprogram.org/wp-content/uploads/2018/03/ Colombia_Presentation.pdf

Ramírez, J. (2014). El turismo en el desarrollo regional: La experiencia de dos departamentos colombianos. Revista Lebret, 6(6), 259-278. Recuperado de https:// dialnet.unirioja.es/descarga/articulo/5983207.pdf 
Fecha de recepción: 31/03/2021 Fecha de aceptación: 01/05/2021 Correspondencia: rosa.gomez@fup.edu.co, navil.mindiola@gmail.com, clau.solarpaz@gmail.com 